\title{
Bauen, Wohnen, Soziale Arbeit
}

\section{Berliner Erfahrungen: Modelle, Konzepte, Kooperationen}

\author{
Clemens Klikar und Dieter Ruhnke
}

\begin{abstract}
A lot of building societies experience that in cases of conflict a high price has to be paid for the lack of communication and understanding of the social situation of their tenants. In addition to court proceedings, tenants move away and there is the danger that houses stay empty. Therefore building societies in Berlin have been establishing for years now a solid cooperation with social organisations.
\end{abstract}

De nombreuses sociétés immobilières savent qu'en cas de conflits, souvent, le prix à payer est lourd en raison d'un manque de communication et d'une faible compréhension de la situation sociale de leurs locataires. En plus des procédures judiciaires, les locataires s'en vont et il y a risque que les logements restent vides. Pour cette raison, les sociétés immobilières de Berlin ont instauré il y a quelques années une coopération stable avec des organisations sociales.

Clemens Klikar, geboren 1961, studierte Architektur und Stadtplanung in Braunschweig und Hamburg. Von 1989 bis 1993 war er Mitarbeiter im Stadtplanungsamt Lemgo, von 1993 bis 2001 im Stadtplanungsamt Göttingen. Seit 2001 leitet er den Geschäftsbereich Stadtentwicklung »Ausnahme \& Regel « bei der Stiftung Sozialpädagogisches Institut Berlin.

Dieter Ruhnke, geboren 1950, studierte Soziologie in Berlin. Von 1980 bis 1989 war er Geschäftsführer des Sozialplanungsbüros BfsS GmbH. Seit 1989 ist er Mitarbeiter, ab 1995 Geschäftsführer der Gesellschaft für Stadtentwicklung, gemeinnützige $\mathrm{GmbH}$.

Internet http://www.stiftung-spi.de
Viele Wohnungsbangesellschaften machen die Erfahrung, dass fehlende Kommunikation und mangelndes soziales Verständnis für die Situation ibrer Mieter im Konfliktfall schnell teuer werden kann. Neben gerichtlichen Auseinandersetzungen drohen Leerstand und Wegzüge. In Berlin gehen Wohnungsbangesellschaften deshalb seit einigen Jabren tragfähige Kooperationen mit sozialen Organisationen ein.

Die Stiftung Sozialpädagogisches Institut Berlin (Stiftung SPI Berlin) und die Gesellschaft für Stadtentwicklung, gemeinnützige $\mathrm{GmbH}$ (GSE gGmbH) kooperieren seit 1982 mit der Wohnungswirtschaft. Sie haben Verwalter-, Geschäftsbesorgungs-, Miet-, Pacht- und Nutzungsverträge abgeschlossen. Als Treuhänder Berlins sind sie mit ihrem Auftrag für all diejenigen, die sich auf dem Wohnungsmarkt nicht selbstständig versorgen konnten, Räumlichkeiten zu akquirieren und zu sichern, der geborene Partner für die Wohnungswirtschaft.

Als » Mitgift « konnten sie früher Mietsicherheiten, Beschäftigungsprogramme und Finanzierungen aus Bauförderprogrammen einbringen. Die Zeiten sind vorbei. Einerseits gibt es kaum noch öffentliche Fördertöpfe, die in die gemeinsamen Projekte eingebracht werden könnten, andererseits haben sich die wohnungswirtschaftlichen Gegebenheiten gründlich gewandelt. Wir stellen daher in diesem Beitrag im Anschluss an die Beschreibung unserer Aufgaben und Kooperationen die Änderung der Rahmenbedingungen dar und skizzieren neue Möglichkeiten der Zusammenarbeit mit der Wohnungswirtschaft.

\section{Wer wir sind}

Die Stiftung Sozialpädagogisches Institut Berlin ist eine gemeinnützige, rechtsfähige Stiftung des bürgerlichen Rechts der Arbeiterwohlfahrt, Landesverband Berlin e. V. Sie ist durch die Satzung den sozial- und gesellschaftspolitischen Leitzielen der Arbeiterwohlfahrt verpflichtet und soll dazu beitragen, »eine Gesellschaft zu entwickeln, in der sich jeder Mensch in Verantwortung für sich und das Gemeinwesen frei entfalten kann «. Über die Art, wie die Stiftung SPI diesen Auftrag umzusetzen hat, fordert der Satzungszweck unmittelbare soziale Praxis und formuliert den Auftrag » zur aktiven Teilnahme an der Lösung sozialer Probleme" und die »Weiterentwicklung von Theorie und Praxis der Sozialarbeit/Sozialpädagogik«. Folgende Konzepte wurden präzisiert und in die Praxis umgesetzt:

- Sicherung von Wohnraum

- Wohnraumbeschaffung für Einkommensschwache, von Wohnungslosigkeit betroffene oder bedrohte Menschen und Klienten der Sozialen Arbeit

- Entwicklung von neuen Finanzierungsund Kooperationsmodellen im sozialen Wohnungsneubau

- Sanierung des Altbaubestandes in Verbindung mit aktiver Selbsthilfe der betroffenen Mieter, Selbsthilfegruppen oder Wohnungssuchenden als Beitrag zur Bekämpfung der Wohnungslosigkeit

- Verbesserung der sozialen Infrastruktur, insbesondere im soziokulturellen Bereich als Kennzeichen einer besseren Lebens- und Wohnqualität der Stadtquartiere, Ausbau und Unterstützung freier Träger

- Erschließen neuer Handlungsfelder für Maßnahmen zur Beschäftigung und beruflichen Qualifizierung von Arbeitslosen

Der Geschäftsbereich Stadtentwicklung "Ausnahme \& Regel « der Stiftung SPI ist als treuhänderischer Sanierungsträger in der Stadterneuerung tätig. Er verbindet Soziale Arbeit und Stadterneuerung in den von ihm betreuten Projekten und ist im Quartiermanagement tätig. Im Rah- 
men der treuhänderischen Sanierungsträgerschaft hat er vier Verfahren der sozialen Stadterneuerung entwickelt und praktiziert:

- das Verfahren der baulichen Selbsthilfe in der Altbaumodermodernisierung mit Selbsthilfegruppen und sozialen Trägern sowie kulturellen Initiativen

- das Treuhandmodell Stiftung SPI-Berlin, Altbausanierung an sozialen Brennpunkten unter aktiver Beteiligung der Mieter und der Schaffung neuer Infrastruktureinrichtung

- Nachbesserung von Großsiedlungen, Wohnumfeldverbesserungsmaßnahmen, Beseitigung baulich-technischer Mängel, Schaffung von mietergetragenen Infrastruktureinrichtungen in diesen Großsiedlungen

- sozialer Wohnungsneubau in Kooperation mit Wohnungsbaugesellschaften unter aktiver Beteiligung freier Träger aus dem Jugend- und Sozialbereich, insbesondere die Schaffung von Wohnraum für Wohnungslose

Die Stiftung SPI hat zur Durchführung der Verfahren die GSE, Gesellschaft für Stadtentwicklung, gemeinnützige $\mathrm{GmbH}$, gegründet. Mehrheitsgesellschafter ist die Stiftung SPI, die anderen Gesellschaftsanteile werden von der Arbeiterwohlfahrt, Landesverband Berlin e. V. gehalten. Laut Gesellschaftervertrag gehören insbesondere folgende Tätigkeiten zum Gegenstand des Unternehmens:

- Die Beschaffung und Verwaltung von Wohn- und Nutzräumen für Personengruppen der Jugendhilfe, sozial benachteiligte Mieter, jugend-, sozial- und sozialkulturelle Projekte sowie deren Beratung und Betreuung

- Organisation, Beratung, Durchführung und Betreuung von baulichen Maßnahmen, einschließlich der Verbesserung des Wohnumfeldes im Rahmen der sozialen Stadterneuerung unter besonderer Berücksichtigung der Förderung der Selbsthilfe im baulichen und sozialen Bereich

- Beschäftigung und berufliche Qualifizierung von Personengruppen aus dem Bereich der Jugendhilfe und Personen aus dem Bereich der Problemgruppen des Arbeitsmarktes mit dem Ziel der dauerhaften sozialen und beruflichen Integration in das Gemeinwesen

Auf Grundlage des zwischen der Gesellschaft für Stadtentwicklung gGmbH (Treuhänder) und dem Land Berlin
(Treugeber) im Jahre 1995 geschlossenen Treuhandvertrages akquiriert und sichert die Gesellschaft für Stadtentwicklung unter sozialen und gemeinnützigen Bestimmungen Räumlichkeiten für die besonderen Bedarfsgruppen der Jugend- und Familienhilfe, der Sozialhilfe und aus dem Gesundheitsbereich sowie für Kulturund Frauenprojekte. Zur Erfüllung ihrer Aufgaben bildet die Gesellschaft für Stadtentwicklung Treuhandvermögen, übernimmt die Verwaltung und soziale Betreuung ganzer Objekte oder tritt als Pächter oder Generalmieter auf.

\section{Eine Wohnung ist mehr als ein Wirtschaftsgut - Kooperationen mit der Wohnungswirtschaft}

Der Treuhandvertrag der Gesellschaft für Stadtentwicklung ist in seinem Auftrage eindeutig und einmalig. Ein wesentlicher Punkt: Wohnraumbeschaffung soll für die Klientel von Trägeren der Jugendund Sozialhilfeträger, für Gesundheitsund Kulturprojekte aus der Position des Vermieters geschehen. Die Gesellschaft für Stadtentwicklung muss also mit denen kooperieren, die über Wohnungen verfügen. In Berlin waren und sind dies im Überwiegenden die ehemals städtischen Wohnungsbaugesellschaften.

Die Gesellschaft für Stadtentwicklung gGmbH bietet der Wohnungswirtschaft Dienstleistungen an, nämlich die Verwaltung ganzer Objekte in Kombination mit sozialer Betreuung. Im Unterschied zum bis dahin üblichen Anmieten von Wohnungen verlangte dies eine neue Profes- sionalität. Termintreue, akkurates Finanz- und Rechtsgebahren, monatliche Abrechnungen und Kontrolle der eigenen Arbeiten waren gefragt.

Die Gesellschaft für Stadtentwicklung als sozialer Träger musste in dieser Kooperation ihre Leistungsstruktur betriebswirtschaftlich rentierlich organisieren, um zu gewährleisten, dass die vertraglich übernommen Aufgaben aus dem vereinbarten Verwalterhonorar finanziert und erledigt werden können. Die neuen Aufgaben waren:

- Vermögensverwaltung

- Übernahme aller Vermieterpflichten

- Wohnungsvergabe

- Abschluss und Kündigung von Mietverträgen

- Mahn- und Klagewesen

- Mieterbetreuung, Mietschuldnerberatung

- bauliche Pflege und Weiterentwicklun der Immobilie

- Erstellen von Wirtschaftsplänen

- Pflege des Wohnumfelds

- Entwicklung und Bewahrung tragfähiger Mieter- und Nachbarschaftsstrukturen

- monatliche Abrechnungen und Rechenschaft

\section{Der Wandel in der kommunalen Wohnungswirtschaft}

Die Ausrichtung der ehemals städtischen und gemeinnützigen Wohnungsbaugesellschaften hat sich in den letzten Jahren gründlich geändert. Die kommunalen Wohnungsbaugesellschaften in

\section{»Ausnahme \& Regel«}

nennt sich der Geschäftsbereich Stadtentwicklung der Stiftung Sozialpädagogisches Institut Berlin (Stiftung SPI Berlin). Er bearbeitet seit seiner Gründung im Jahre 1981 unterschiedliche Aufgaben der Stadterneuerung. Insbesondere die Klientel der Jugend- und Sozialhilfe, wirtschaftlich in Not geratenen Personen, arbeitslosen Jugendlichen, jungen Erwachsenen und Langzeitarbeitslosen, den freien Trägern der Jugend- und Sozialhilfe sowie soziokulturellen Trägern ist die Stiftung SPI in ihrer Arbeit verpflichtet.

In seiner Zielsetzung ist der Geschäftsbereich Stadtentwicklung ausgerichtet auf besondere soziale und stadtpolitische Problemstellungen, die Sicherung sozialer Standards, die Integration von sozial und wirtschaftlich in Not geratenen oder strukturell benachteiligten Bevölkerungsgruppen, ihre Versorgung mit Wohnraum, das Sichern preiswerten Wohnraums, ihre Erwerbstätigkeit und berufliche Qualifizierung sowie ihre Teilhabe am sozialen und kulturellen Leben.

Stiftung Sozialpädagogisches Institut, Geschäftsbereich Stadtentwicklung, Treuhänderischer Sanierungsträger Ausnabme \& Regel, Prinzenallee 74, 13357 Berlin (Wedding), Telefon 030 493001-10, Fax 030 493001-12, E-Mail ausnabme.regel@stiftung-spi.de, Internet http://www.stiftung-spi.de 
Berlin hatten bis vor wenigen Jahren neben ihrer wohnungspolitischen Grundaufgabe, Daseinsvorsorge für ein breites Bevölkerungsspektrum abzusichern, die Aufgabe Stadterneuerung in Altbauquartieren zu betreiben, Plattenbauwohnungen zu sanieren und Wohnungen neu zu bauen.

In Zeiten angespannter Wohnungsmarktsituation schon Ende der 1980er Jahre empfanden die Wohnungsbaugesellschaften die immerwährenden Forderungen nach Wohnungen aus dem Bereich der Jugend- und Familienhilfe sowie der Sozialpolitik als anmaßend und unseriös. In der Massierung von sogenannten Problemfällen in ihrem Bestand ohne eine verbindliche begleitende Hilfsstellung, Betreuung und Beratung, sehen sie eine Gefährdung ihrer wohnungswirtschaftlichen Gesamtaufgabe. Vielfach bekamen sie mit dem zur Verfügung stellen von Wohnraum für die Klientel der Jugend und Familienhilfe auch die sozialen Folgenprobleme übertragen. Mit deren Bearbeitung waren und sind sie überfordert, da sie keinen Apparat dafür haben und ihr öffentlicher Auftrag das auch nicht abdeckt.

Der Wohnungsmarkt entwickelte sich in den 1990er Jahre anders als vorausgesagt. Das allseits prognostizierte Bevölkerungswachstum für Berlin fand nicht statt. Die Wohnungsbaugesellschaften blieben erstmalig auf ihren Wohnungen sitzen. Die Mieter konnten wählen. Die Wohnungsbaugesellschaften mussten um die Mieter buhlen.

Die öffentlich geförderte Altbausanierung und der soziale Wohnungsbau und seine Folgefinanzierung wurden eingestellt. Dadurch kamen zusätzliche finanzielle Lasten auf die Wohnungsbaugesellschaften zu. Das Land als Eigentümer, das durch Defizite seiner Gesellschaften belastet worden wäre, stärkte seine $\mathrm{Ge}$ sellschaften durch Zusammenschlüsse und entlastete sich von finanziellen Risiken durch Verkäufe von attraktiven Teilbeständen. In den Jahren 2004 und 2005 wurden bundesweit 550.000 ehemals kommunal kontrollierte Wohnungen privatisiert. Das Land Berlin verkaufte 2004 seine größte Wohnungsbaugesellschaft mit 70.000 Wohneinheiten. Dieser Trend zum Verkauf hält an.

Die Zielsetzung der kommunalen Wohnungswirtschaft musste sich ändern: Nicht mehr Wohnungsbewirtschaftung zur Daseinsvorsorge, schon gar nicht Wohnraumbewirtschaftung für die besonderen Bedarfsgruppen des Wohnungsmarktes, sondern Wohnungswirtschaft ohne öffentliche Gelder und Subventionen bis hin zur Wohnungswirtschaft zum Handeln mit Wohnungen, wurde das Motto. Stabilisierung und Optimierung der Finanzlage durch Effektivierung des Personaleinsatzes, Delegierung von Regelaufgaben an Fremdfirmen und das Abstoßen von nichtrentablen Wohnungen wurden zu zentralen Aufgaben der Gesellschaften.

\section{Der Wandel bei den sozialen Trägern}

Mit der Einheit von Berlin und dem Aufbau der Trägerstrukturen im Gesamt-
Ganz praktisch hatte dies beispielsweise Auswirkungen auf die Träger von betreutem Jugendwohnen. Die Wohnungen für betreutes Einzelwohnen sollten wegen der Effektivität des Personaleinsatzes räumlich eng beieinander liegen. Das war und ist kaum zu realisieren. Die Entspannung auf dem Wohnungsmarkt hatte in diesem Zusammenhang zu keiner Verbesserung ihrer Versorgung geführt: Kleine und preiswerte Wohnungen sind bis heute weiterhin Mangelware.

Erschwerend kam hinzu, dass das Landesprogramm zur Förderung von wohnungspolitischen Projekten eingestellt und damit den Trägern ein wesentliches Standbein bei der Initiierung neuer Projekte genommen wurde.

Zusätzlich wurden die Träger geschwächt durch die Umorientierung von Beschäftigungs- und Qualifizierungsmaß-

\section{"Was wir Wohnungsgesellschaften bieten: Verwaltung ganzer Objekte in Kombination mit sozialer Betreuung «}

bereich kam es zu einer Wohnungsnachfrage von Trägern der Jugend- und Sozialhilfe, die kaum zu befriedigen war. Die Gesellschaft für Stadtentwicklung als Verwalter, aber auch Generalmieter oder Pächter von Wohnungen oder ganzen Häusern, sicherte den Wohnungsbaugesellschaften die monatliche Miete und stellte die Wohnungen den Trägern zur Verfügung. Zudem wurden mit und für die Träger Wohnungen und Häuser unter Zuhilfenahme von öffentlichen Mitteln aus dem Selbsthilfeprogramm des Landes Berlin und mit Einsatz von Beschäftigungsmaßnahmen der zuständigen Arbeitsämter ausgebaut und hergerichtet.

Der Prozess der Ökonomisierung der Sozialen Arbeit in unserer Gesellschaft hat in den letzten Jahren deutlichen Einfluss auf die sozialen Träger genommen. Relativ zügig bildete sich ein Markt der Sozialen Arbeit, auf dem sie untereinander als Dienstleister in Konkurrenz standen. Sie waren gefordert Angebote vorzulegen, die qualitativ und preislich überzeugend sind und sie unterliegen mit ihren Leistungen und der Qualität ihres eingesetzten Personals der Rechenschaft und Kontrolle. nahmen. Diese konnten bis Ende der 1990er Jahre relativ problemlos eingesetzt werden. Die Einsatzmöglichkeiten wurden jedoch in den letzten Jahren drastisch verändert. Keine Regelaufgaben, nur noch zusätzliche Bereiche werden finanziert. Zudem wurden Kontingente, Fristen und Dauer von Maßnahmen erheblich reduziert.

\section{»Rien ne va plus«}

...könnte man meinen. Die Wohnungsbaugesellschaften reduzieren sich auf ihr Kerngeschäft und die sozialen Träger fristen mit ein bisschen Glück in Nischen ihr Dasein.

Die reale Situation sieht allerdings nicht ganz so traurig aus. Den Chancen der Privatisierung kommunaler Wohnungsbestände wie Wiedergewinnung kommunaler Handlungs- und Finanzierungsspielräume, Steigerung der Bewirtschaftungseffektivität und Aufwertung von Beständen, stehen die Risiken gegenüber, beispielsweise Preissteigerungen, soziale Verdrängung und Vernachlässigung von Beständen, die aufgrund von Lage und 
Zustand nicht verwertbar sind. Hier muss flankierendes Handeln ansetzen, hier können soziale Träger ihren Platz in der Kooperation mit der Wohnungswirtschaft finden.

\section{Neuausrichtung von Wohnungsbewirtschaftung}

Um das "Wirtschaftsgut Immobilie « erfolgreich mittel- oder langfristig gestalten und verwerten zu können, liegt es im Interesse der Wohnungsbaugesellschaften, an einer sozialen Stabilisierung ihrer Quartiere im Sinne einer sozialen Stadterhaltung mitzuwirken.
Nicht alle Wohnungen können verkauft werden, schon gar nicht alle auf einmal. Ein Grundstock von Wohnungen wird und soll auch zukünftig von den städtischen Gesellschaften gehalten werden; zum einem als strategische Reserve zum renditeorientierten Bewirtschaften und zum anderen zur Wohnraumversorgung von sozial schwachen Haushalten und all den Gruppen, die sich nicht selbstständig auf dem Wohnungsmarkt versorgen können.

Die Bewirtschaftung dieser Bestände verlangt neben der technischen und baulichen Pflege präventives Handeln zur Stabilisierung der Mieterstruktur, Beteiligungsmodelle für die Mieter sowie Be- treuungsstrukturen und Betreuungsangebote. Die Mieter sollen als Kunden gehalten werden, in ihrem Kiez wohnen bleiben. Dazu müssen Wohnungsstandard, Wohnungszuschnitt und Instandhaltungszustand den modernen Erfordernissen gerecht und kundenorientiert individuell angepasst werden sowie das Wohnumfeld ansprechend gestaltet worden, ohne die Mietzahlungsfähigkeit der Bewohner zu überfordern.

In der nötigen Kundenorientierung und für den Umgang mir den Mietern zur Erreichung der Ziele sind Qualifikationen gefragt, die üblicherweise in einer auf ihr Kerngeschäft ausgerichteten Wohnungsbaugesellschaft nicht vorhanden sind -

\section{Kooperationen: So kann es gehen}

Die schlechten Erfahrungen mit ungenügender Kommunikation und fehlender sozialer Betreuung ihrer Mieter veranlasste eine Berliner Wohnungsbaugesellschaft zum Umdenken: In einer Siedlung der 1960er Jahre im Süden der Hauptstadt standen grundlegende Instandsetzungen an. Die Mieter, überwiegend noch die Erstbezugsmieter, sind im Rentenalter. Die technische Ausrüstung der Gebäude ist marode und erneuerungsbedürftig. Der bauliche Aufwand in den Wohnungen ist dadurch enorm: Die Küche und das Bad werden zu Komplettbaustellen und während der Bauzeit nicht nutzbar.

Die Instandsetzungsmaßnahmen werden aus dem Etat der Wohnungsbaugesellschaft finanziert, da öffentliche Mittel nicht zur Verfügung stehen. Die Pflicht ein öffentliches Sozialplanverfahren und Mieterbetreuung anzubieten, entfiel damit für die Wohnungsbaugesellschaft.

Sie entschied sich auf eigene Kosten eine Mieterbetreuung durchzuführen, um mit einem sozial verträglichen und zügigen Verfahren mit intensiver Information und Mieterbeteiligung einen reibungslosen und damit kostengünstigen Bauablauf zur Herstellung zeitgemäßer Wohnverhältnisse zu gewährleisten. Die Mieterbetreuung wurde beschränkt ausgeschrieben und das folgende »Leistungsverzeichnis Mieterbetreuung « vereinbart:

\section{Phase I - Maßnahmefeststellung}

- Teilnahme an allen für die Durchführung der Maßnahme notwendigen Vorgesprächen

- Abstimmung aller mieterrelevanten Regelungen mit dem Bauherrn

- Organisation und Einladung zu Mieterversammlungen, anschließende Auswertung mit dem Bauherrn

- kontinuierliche Telefonauskunft für Mieteranfragen

- Einzelgespräche mit allen Mietern zur Feststellung sozialer Härtefälle und zur Mitwirkungsbereitschaft der Mieter zur Erörterung und zum Anbieten von geeigneten Hilfsmaßnahmen, zur Darlegung der Baumaßnahmen und der Baudurchführung darzulegen, zum Abschluss von Durchführungsvereinbarungen

\section{Phase II - Baudurchführung}

- Durchführung wöchentlicher Sprechstunden

- Beratung und Information, Konfliktlösungsangebote

- Veranlassung von finanziellen und sonstigen Hilfsmaßnahmen, Entschädigungen, Umzugs-, Putz- und Kochhilfen, Räumen und Rücken, De- und Remontagearbeiten etc.

- Teilnahme an Baubesprechungen, Abstimmung mit den an der Baumaßnahme Beteiligten

\section{Härtefälle}

Für Fälle mit erhöhtem Betreuungsaufwand und nötiger Einzelfallbetreuung wegen besonderer Hilfebedürftigkeit wurde folgendes Profil aufgestellt:

- besondere körperliche, gesundheitliche oder altersbedingte Schwierigkeiten

- besonders schwierige soziale und/oder finanzielle Lebenslagen

- sprachliche Barrieren bei ausländischen Mietern

- Verweigerung der Maßnahme

- Unauffindbarkeit

- nötige Fremdunterbringung während der Baumaßnahme

\section{Finanzierung}

Der Standort für die Mieterbetreuung wird mit nötiger technischer Infrastruktur von der Wohnungsgesellschaft kostenlos zu Verfügung gestellt. Der Aufwendungsersatz für die Gesellschaft für Stadtentwicklung wird durch einen vereinbarten Stundensatz geleistet. Für Härtefälle gab es einen Zuschlag. Die Leistungen werden monatlich mit prüffähigen Leistungsnachweisen in Rechnung gestellt.

\section{Kooperationen}

Die Betreuungstätigkeit verlangt neben der engen Zusammenarbeit mit dem Bauherrn und den von ihm beauftragten Firmen ein umfassendes Spektrum an Kontaktaufnahme und Kooperation mit Verwandten, Sozialstationen, Kranken- und Pflegekassen, Sozialund Jugend- und Arbeitsämtern, Mieterbeiräten, Umzugsfirmen, Pensions- und Ferienwohnungsbetreibern, Seniorenwohnhäusern, Mietschuldnerberatungsstellen, Sicherheitsdiensten, Haushaltshilfen, Wohngeldämtern etc. Clemens Klikar und Dieter Rubnke 
die Mitarbeiterschaft der Wohnungsbaugesellschaften setzt sich im Wesentlichen aus Kaufleuten der Wohnungswirtschaft, Technikern und Betriebswirten zusammensetzt. Neue Kooperationen bieten sich an.

\section{Mieterbetreuung im Auftrag einer Wohnungsbaugesellschaft}

Bis zu 200.000 Wohneinheiten sollen in Berlin zur Sicherung der Wohnungsversorgung im Bestand der ehemals städtischen Wohnungsbaugesellschaften verbleiben. Bestandspflege von Wohnungen aus eigenen Mitteln und nach einem eigenen Wirtschaftsplan wurde zu einem wichtigen Bestandteil ihrer Arbeit.

Praktisch heißt das: Instandsetzung und Wohnungsverbesserung bei bewohntem Zustand ohne öffentlich finanzierte Sozialpläne und Entschädigungsleistun- so in einer Siedlung im Süden Berlins, die vor 40 Jahren errichtet wurde (vgl. Kasten »Kooperationen: So kann es gehen «).

\section{Fazit}

Ein dauerhafter Kooperationsverbund von Wohnungswirtschaft und Sozialer Arbeit ist gerade in den Zeiten neuer Armut und sozialer Segregation eine erfolgversprechende Zukunftsstrategie: Die Mieter und Bürger werden mit ihrer Entscheidungskompetenz eingebunden in wesentliche Entscheidungsprozesse bei der Gestaltung ihrer persönlichen Wohnsituation.

Der Erfolg unserer Kooperation mit der Wohnungswirtschaft spricht für sich:

- wenig Auszüge aus den Häusern

- Bewahrung und Verbesserung der nachbarschaftlichen Verhältnisse

\section{"Was wir garantieren müssen:}

\section{Termintreue, akkurates Finanz-und \\ Rechtsgebabren, monatliche Abrechnungen, Kontrolle der eigenen Arbeiten "}

gen für die Mieter. Es gelten die vertraglichen Regelungen der Mietverträge und der Mietengesetzgebung.

Wie sich gezeigt hat, hat die Reduzierung der Kommunikation der Wohnungsbaugesellschaften mit den Mietern in diesen Angelegenheiten einzig auf diese Ebene des Mietrechts den Mietern erstaunlich viele Widerspruchsmöglichkeiten ermöglicht. Ganz zu schweigen von Bauverzögerungen durch Informationspannen: Mieter waren nicht anwesend, Handwerker konnten nicht in die Wohnung etc. Im Ergebnis gab es einerseits gestiegene Baukosten, andererseits Unzufriedenheit und Ärger auf Mieterseite. Wegzüge drohten. Durch teure Instandsetzungen Leerstand produzieren - das kann sich in Zeiten entspannter Wohnungsmärkte kein Vermieter leisten.

In später folgenden Quartieren, in denen grundlegende Instandsetzungen anstanden, sollte es sozial verträglicher und baulich reibungsloser organisiert werden,
- Beteiligung der Mieter durch Mieterbeiräte

- zusätzliche Betreuungsangebote von Sozialstationen

- Initiierung von Bürgertreffs, Geselligkeit, Tauschbörsen etc.

Die Mitwirkung bei Planung und Durchführung fördert die Aneignung der Wohnung und des Wohnumfeldes und damit den verantwortungsvollen und sorgfältigen Umgang. Die Mieterstruktur bleibt stabil, Fluktuation wird reduziert. Alte Mieter bleiben durch altersgemäße Wohnungsanpassung länger als bisher üblich selbstständig in ihren Wohnungen. Isolation und Abkapselung können tendenziell aufgebrochen werden. Nachbarschaften werden gestärkt und Hilfsbereitschaft gefördert. Betreuungsangebote können sich im Nahbereich erfolgreich ansiedeln.

\section{Aktuelle \\ Neuerscheinung}

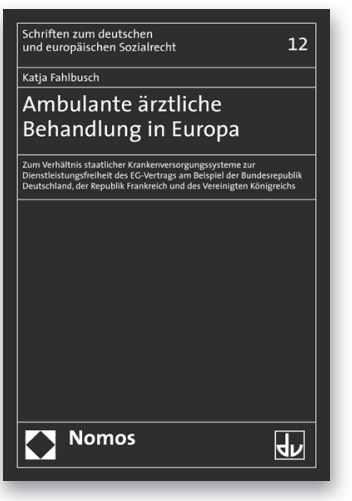

\section{Ambulante ärztliche Behandlung in Europa}

Zum Verhältnis staatlicher Krankenversorgungssysteme zur Dienstleistungsfreiheit des EG-Vertrags am Beispiel der Bundesrepublik Deutschland, der Republik Frankreich und des Vereinigten Königreichs Von Dr. Katja Fahlbusch 2006, 398 S., brosch., 49,- $€$, ISBN 978-3-8329-1956-6

(Schriftenreihe zum deutschen und europäischen Sozialrecht, $B d .12$ )

Im Spannungsfeld nationaler Krankenversorgungssysteme zum Europarecht widmet sich diese Arbeit dem Aspekt ambulanter ärztlicher Versorgung.

Auf der Grundlage der Darstellung der rechtlichen Grundlagen in Deutschland, Frankreich und dem Vereinigten Königreich werden die Vereinbarkeit mit der Dienstleistungsfreiheit geprüft und Gestaltungsvorschläge gemacht.

\section{Nomos}

